\title{
Is forced expiratory volume in six seconds a valid alternative to forced vital capacity?
}

\section{To the Editors:}

We have read with interest the article by HANSEN et al. [1] in a recent issue of the European Respiratory Journal, stating that the use of forced expiratory volume in six seconds (FEV6) reduces the sensitivity of obstructive lung disease detection. Their study population of 3,515 current smokers, with a $17.5 \%$ prevalence of obstruction, found a sensitivity of only $76.8 \%$ and a specificity of $98.2 \%$ when comparing FEV1/FEV6 with FEV1/forced vital capacity (FVC) below their respective 95\% lower limits of normal (LLN). Based on this low sensitivity, HANSEN et al. [1] concluded that FEV1/FEV6 is less reliable than FEV1/FVC as a screening parameter for the detection of obstruction.

The lower sensitivity obtained by HANSEN et al. [1] with respect to previous studies, in which sensitivity typically ranged between $92-95 \%$ [2-5], was attributed to differences in study population. HANSEN et al. [1] contended that previous studies comparing the performance of FEV6 and FVC in detecting obstruction [3-5] had too few mildly obstructive and normal subjects. However, HANSEN et al. [1] cite another study, by AKPINAR-ELCI et al. [2], which showed 92\% sensitivity and $98 \%$ specificity for the detection of obstruction; this is despite a prevalence of obstruction of only $14.5 \%$ in 1,139 nonpatients in a workplace setting. In general, these participants are healthy, and obviously median spirometry values are closer to the LLN than for groups of symptomatic patients referred to a pulmonary function laboratory.

In the study by HANSEN et al. [1], the "minimal obstructed" group represents the largest group (59\%) of all subjects considered obstructive. Minimal obstruction was defined as FEV1/FVC and/or FEV3/FVC below the 95\% LLN, combined with FEV1 between $80-120 \%$ predicted. However, the rationale for using this criterion of possible morbidity only holds in populations with respiratory diseases; for healthy subjects, the meaning of a low FEV1/FVC with a normal FEV1 is unclear [6]. The proportion of subjects with respiratory diseases studied by HANSEN et al. [1] is not explicitly mentioned, but is likely to be small. Therefore, a substantial proportion of subjects labelled with "minimal obstruction" could be normal. Figure 3 in the paper by HANSEN et al. [1] clearly shows that the number of false negatives in the "minimal obstructed" group represents more than half of all false negatives; this could introduce a serious bias to the results.

In previous studies by us and others [2-5], most of the discordant cases had FEV1/FVC and FEV1/FEV6 values close to their respective $L L N$, i.e. in a range where diagnosis of obstruction is questionable. Values close to the LLN can be assumed from table 5 in the paper by HANSEN et al. [1], because the mean deviation is only $2.64 \%$. Considering the day-to-day variability of spirometry, the minimally obstructive subjects could easily shift between the normal and obstruction categories on different testing occasions. Therefore, one could question the clinical relevance of detecting borderline obstruction.

In addition, we should be careful when comparing two diagnostic tests with each other in the absence of a true gold standard. In reality, FEV1/FVC is more of a "de facto standard", and by using it as a gold standard for the detection of obstruction, FEV6 can never be shown to outperform FVC in the denominator of this ratio to better reflect true airway obstruction. This is particularly true when studying a population with FEV1/FVC and FEV1/FEV6 values close to their respective LLN.

The increasing difference between FEV6 and FVC volumes with increasing age and severity of obstruction is probably irrelevant in terms of identifying airway obstruction, because the deficit in airflow will be present long before $6 \mathrm{~s}$. Moreover, spirometric detection of airway obstruction is a matter of categorisation and not of quantitative agreement. As long as FEV1/FVC and FEV1/FEV6 values stay on the same side of their respective cut-off points the absolute values of these ratios are of little clinical importance, because once obstruction is established, the severity of this obstruction will be evaluated using FEV1 [6].

In conclusion, the main advantage of using forced expiratory volume in six seconds values in primary care is to assist with the quality of testing. Forced expiratory volume in six seconds is more reliable than forced vital capacity, and using the $6 \mathrm{~s}$ cut-off eliminates the need for prolonged exhalations, eliminates the need to meet reproducible forced vital capacity values, and is an easier test for patients to perform. In practice, the advantages of using forced expiratory volume in six seconds values may well more than compensate for the small differences in classification of patients' results that are clustered around the lower limits of normal.

\section{J. Vandevoorde* and M. Swanney ${ }^{*}$}

*Dept General Practice, University of Brussels (Vrije Universiteit Brussel), Brussels, Belgium, and "Respiratory Physiology Laboratory, Christchurch Hospital, Christchurch, New Zealand.

\section{REFERENCES}

1 Hansen JE, Sun X-G, Wasserman K. Should forced expiratory volume in six seconds replace forced vital capacity to detect airway obstruction? Eur Respir J 2006; 27: 1244-1250.

2 Akpinar-Elci M, Fedan KB, Enright PL. FEV6 as a surrogate for FVC in detecting airways obstruction and restriction in the workplace. Eur Respir J 2006; 27: 374-377.

3 Swanney MP, Jensen RL, Crichton DA, Beckert LE, Cardno LA, Crapo RO. FEV6 is an acceptable surrogate for 
FVC in the spirometric diagnosis of airway obstruction and restriction. Am J Respir Crit Care Med 2000; 162: 917-919.

4 Vandevoorde J, Verbanck S, Schuermans D, Kartounian J, Vincken W. FEV1/FEV6 and FEV6 as an alternative for FEV1/FVC and FVC in the spirometric detection of airway obstruction and restriction. Chest 2005; 127: 1560-1564.

5 Vandevoorde J, Verbanck S, Schuermans D, Kartounian J, Vincken W. Obstructive and restrictive spirometric patterns: fixed cutoffs for FEV1/FEV6 and FEV6. Eur Respir J 2006; 27: 378-383.

6 Pellegrino R, Viegi G, Brusasco V, et al. Interpretative strategies for lung function tests. Eur Respir J 2005; 26: 948-968.

DOI: $10.1183 / 09031936.00100206$

\section{From the authors:}

We welcome the comments of J. Vandevoorde and $\mathrm{M}$. Swanney, who have been at the forefront of advocacy for the substitution of forced expiratory volume in six seconds (FEV6) and its ratios for forced vital capacity (FVC) and its ratios. While we respect their findings, our study [1] concerned the use of spirometry to detect airway obstruction in a relatively healthy population, the third National Health and Nutrition Evaluation Survey (NHANES-III), rather than its clinical use in following patients with known disease.

We hope we agree that in following patients with known and significant airway obstruction or in assessing responsiveness to inhaled bronchodilators, the absolute values of FEV1 or FEV3 are optimal, and ratios such as $\mathrm{FEV} 1 / \mathrm{FEV} 6, \mathrm{FEV} 1 / \mathrm{FVC}$ or mean forced expiratory flow between 25 and $75 \%$ of $\mathrm{FVC}$ (FEF25-75\%) are not. In following these patients, there is no need for repeated lengthy forced manoeuvres, such as FEV6 or FVC. Unforced vital capacities not only suffice, but may be more informative and less stressful than repeated FEV6 or FVC manoeuvres.

The question of selecting spirometric values to detect early airway obstruction, especially in primary-care settings, which was our focus, is more complex. For detecting obstruction, adding a measurement that focuses on the proportional increase in long time-constant lung units, i.e. the $\mathrm{FEV}_{3} / \mathrm{FVC}$ (or 1-FEV3/FVC), supplements the FEV1/FVC well. J. Vandevoorde and M. Swanney seem to object to us adding the FEV3/FVC as a measure to detect obstruction in "healthy subjects", yet the NHANES-III smokers clearly had greater increases of long time-constant lung units (1-FEV3/FVC) than nonsmokers [1]. This increase may be undetected by the FEV1/ FEV6 measurement, while the FEF25-75\%, using either the FEV6 or FVC as a denominator, is certainly not sufficient to independently detect airway obstruction in a statistically valid way in a relatively healthy population [2].

One could question the clinical relevance of detecting borderline obstruction, except for epidemiological studies and for the purpose of the early detection of developing disease. In fact, another proponent of the FEV6 measurement editorialises that the screening for chronic obstructive pulmonary disease by primary-care physicians has the potential to do more harm than good [3]. However, we think that early detection of airway obstruction and identification of the cause have merit. Committee guidelines from authorities for detecting airway obstruction are useful [4], but not necessarily the last word, as evidenced by recent public correspondence [5,6] and the conflicts over the Global Initiative for Chronic Obstructive Lung Disease standard of FEV1/FVC $<70 \%$ to define airway obstruction $[4,6]$.

In many, if not all, studies [2, 7-9], the coefficients of variation for the FEV1/FVC and FEV3/FVC are much lower than for FEV1, FEV3, FVC or FEF25-75\%. We contended that airway obstruction was likely to be present if either the FEV1/FVC and/or $\mathrm{FEV} 3 / \mathrm{FVC}$ before bronchodilator administration were $<95 \%$ confidence levels, even though the absolute FEV1 may have been $>80 \%$ mean predicted. Thus, in detecting, rather than quantifying obstruction, the spirometric curve after $1 \mathrm{~s}$, and even after $6 \mathrm{~s}$, may be important. Ignoring measurements that detect the increase in long time-constant airspaces lessens the ability to detect early disease. Therefore, in detecting airway obstruction, volume ratios such as FEV1/FVC and $\mathrm{FEV}_{3} / \mathrm{FVC}$ should both be useful. In contrast, absolute values, such as FEV1 and FEV3, are valuable in quantifying obstruction or assessing response to bronchodilators or to follow the progress of known disease.

Clinically, when spirometric values are borderline, clinical evaluation including a good history and physical examination, bronchodilator testing, and measurement of gas-transfer index may be necessary to diagnose or exclude lung disease. Neither ignoring airway obstruction nor over-diagnosis and overtreatment are desirable. However, let us acknowledge that spirometry has three major uses: 1) detection of airway obstruction; 2) assessment of disease progression; and 3) assessment of therapy. For the first, we showed that values of both the FEV1/FVC and FEV3/FVC separated NHANES-III current smokers from never-smokers by $\sim 20$ yrs by middle age [2]. We did not assess the similar effectiveness of the FEV3/ FEV6, the progression of disease and therapy. However, for assessing disease progression or effect of therapy, we stress that the absolute values of FEV1 and FEV3 should be more sensitive than any ratios, including FEV1/FEV6, FEV1/FVC or FEF25-75\%, because both numerator and denominator may increase or decrease together, thereby obscuring the absolute change in the fast time-constant lung units (those that contribute to the flow during the first second).

We hope that we can find common ground with J. Vandevoorde and M. Swanney and their colleagues in recommending that spirometry to detect airway obstruction should be performed on good equipment by dedicated, trained and experienced technicians, under the supervision of physicians experienced and skilled in the interpretation of spirometry. We also believe that optimal spirometry measurements to detect early airway disease should include detection of an abnormal increase in proportion of longer time-constant (diseased) lung units. Conversely, absolute timed volumes, rather than ratios, are useful for following patients with known disease.

\section{J. Hansen, X-G. Sun and K. Wasserman}

Harbor-University of California, Medical Center, Torrance, CA, USA. 